\title{
Analysis of 142 genes resolves the rapid diversification of the rice genus \\ Xin-Hui Zou ${ }^{\alpha^{*}}$, Fu-Min Zhang ${ }^{\varkappa^{*}}$, Jian-Guo Zhang ${ }^{\star \dagger}$, Li-Li Zang*, Liang Tang*, Jun Wang ${ }^{\dagger}$, Tao Sang ${ }^{\ddagger}$ and Song Ge*§
}

Addresses: *State Key Laboratory of Systematic and Evolutionary Botany, Institute of Botany, Chinese Academy of Sciences, Beijing, 100093, China. ${ }^{\dagger}$ Beijing Genomics Institute, Beijing, 101300, China. ${ }^{*}$ Department of Plant Biology, Michigan State University, East Lansing, MI 48824, USA. §The Graduate School, Chinese Academy of Sciences, Beijing, 100039, China.

$\ltimes$ These authors contributed equally to this work.

Correspondence: Song Ge. Email: gesong@ibcas.ac.cn

Published: 3 March 2008

Genome Biology 2008, 9:R49 (doi: 10.1 I 86/gb-2008-9-3-r49)

The electronic version of this article is the complete one and can be found online at http://genomebiology.com/2008/9/3/R49
Received: 21 December 2007

Revised: 18 February 2008

Accepted: 3 March 2008

(c) 2008 Zou et al.; licensee BioMed Central Ltd.

This is an open access article distributed under the terms of the Creative Commons Attribution License (http://creativecommons.org/licenses/by/2.0), which permits unrestricted use, distribution, and reproduction in any medium, provided the original work is properly cited.

\begin{abstract}
Background: The completion of rice genome sequencing has made rice and its wild relatives an attractive system for biological studies. Despite great efforts, phylogenetic relationships among genome types and species in the rice genus have not been fully resolved. To take full advantage of rice genome resources for biological research and rice breeding, we will benefit from the availability of a robust phylogeny of the rice genus.

Results: Through screening rice genome sequences, we sampled and sequenced 142 single-copy genes to clarify the relationships among all diploid genome types of the rice genus. The analysis identified two short internal branches around which most previous phylogenetic inconsistency emerged. These represent two episodes of rapid speciation that occurred approximately 5 and 10 million years ago (Mya) and gave rise to almost the entire diversity of the genus. The known chromosomal distribution of the sampled genes allowed the documentation of whole-genome sorting of ancestral alleles during the rapid speciation, which was responsible primarily for extensive incongruence between gene phylogenies and persisting phylogenetic ambiguity in the genus. Random sample analysis showed that 120 genes with an average length of 874 bp were needed to resolve both short branches with $95 \%$ confidence.

Conclusion: Our phylogenomic analysis successfully resolved the phylogeny of rice genome types, which lays a solid foundation for comparative and functional genomic studies of rice and its relatives. This study also highlights that organismal genomes might be mosaics of conflicting genealogies because of rapid speciation and demonstrates the power of phylogenomics in the reconstruction of rapid diversification.
\end{abstract}




\section{Background}

Rice is one of the most important crops in the world, providing the staple food for more than one-half of the world's population $[1,2]$. The completion of rice genome sequencing has made rice and its wild relatives an increasingly attractive system for biological studies at the genomic level [3-5]. Considerable insights have been recently gained into comparative genomics between rice and other cereal crops of the grass family [6] and between the species of the rice genus, Oryza $[7,8]$. To take full advantage of rice genome resources for basic biological research and rice breeding, we will benefit from the availability of a robust phylogeny of the rice genus.

The genus Oryza consists of 2 cultivated and approximately 22 wild species distributed in a diverse range of habitats in tropics and subtropics of the world [9]. By assessing the degree of meiotic pairing in interspecific hybrids, traditional genome analyses grouped the majority of Oryza species into five diploid and two allotetraploid genome types: A-, B-, C-, E-, F-, BC-, and CD-genomes [10]. Because of the difficulties in obtaining hybrids with presumably more distantly related species, three additional genomes, G-, HJ-, and HK-genomes, were later recognized based on total genomic DNA hybridization [11] and molecular phylogenetics [12]. In Oryza, onethird of extant species are allotetraploids that originated through hybridization between diploid genomes, and, in particular, four (B-, E-, F-, and G-genomes) out of the six diploid genomes each have a single species $[1,9,13]$. Consequently, elucidating the phylogenetic relationships of the diploid rice genomes is critically important for understanding the evolutionary history of the entire genus.

Despite extensive studies on evolutionary relationships among rice genomes and species [10,12,14-16], the phylogenetic relationships among genomes remained elusive until a study that sampled all recognized Oryza species and utilized sequences of two nuclear and one chloroplast genes [12]. This study supported the monophyly of each of the previously recognized genome types and reconstructed the origins of tetraploid species. Nevertheless, two areas of the phylogeny were left unresolved due to incongruence between gene trees. These included the relationship among A-, B-, and Cgenomes and that among the F-genome, G-genome, and the rest of the genus [12]. The incongruence was highlighted in the rice phylogenetic literature, where all three possible relationships among A-, B-, and C-genomes were suggested [10,12,16-18]. More remarkable is the position of the Fgenome, which varied from being the most basal lineage of the entire genus $[16,19]$ to being nested within the recently diverged A-genome $[15,20]$.

The recent decade has witnessed the successful utilization of large quantities of DNA sequences in solving long-standing phylogenetic problems [21-30]. As a growing number of genomes are decoded, phylogenetic reconstruction using genome-wide markers, or phylogenomics [31,32], will pro- vide unprecedented opportunities to elucidate the previously controversial evolutionary relationships at all taxonomic levels [31,33]. In this study, we screened the genome sequences of two rice cultivars and sampled 142 single-copy genes as markers for reconstructing the phylogeny of all diploid rice genomes. This phylogenomic analysis, for the first time, fully resolved the relationships of the rice genome types. It further revealed that two episodes of rapid diversification in the rice genus were responsible for the phylogenetic incongruence that persisted in the previous studies. We suggest that rapid diversification might be widespread in organismal evolution and caution that under rapid speciation, large data sets or phylogenomic approach are required to resolve phylogenetic relationships with a high degree of confidence.

\section{Results \\ Phylogeny inferred from concatenated sequences of 142 genes}

After an extensive screen of rice genome sequences, we identified and sequenced 142 single-copy genes that were most likely free of the paralogy problem for reconstructing the phylogeny of all diploid genome types of Oryza (Table 1; see Materials and methods for details of gene screening). These genes are distributed throughout the 12 rice chromosomes and represent a genome-wide sampling of phylogenetic markers (Additional data files 1 and 2). After removing regions with ambiguous alignment, we concatenated the 142 genes into a data matrix of $124,079 \mathrm{bp}$, with exons accounting for $43 \%$ of the total sequence. The concatenated alignment contained 26,838 (21.6\%) variable sites, of which 6,753 (5.4\%) were phylogenetically informative (Additional data file 2).

Phylogenetic analyses of the concatenated sequences using maximum likelihood (ML), maximum parsimony (MP) and Bayesian inference (BI) all yielded a single fully resolved tree with high bootstrap support or Bayesian posterior probability (PP) for all internal branches (Figure 1). We labeled these branches as I, II, III, and IV. The relationships between A-, B-, and C-genomes are finally resolved, with the sister relationship between A- and B-genomes supported by 99-100\% bootstrap support or PP. The F-genome, which jumped all over the previously reported phylogenies, is firmly placed between the basal G-genome and the rest of the genome types.

Because the increase in sequence length or the number of sampled genes does not guarantee the elimination of systematic errors $[28,34,35]$, it is necessary to investigate the potential impact of systematic bias on our phylogenetic reconstruction. First, we tested homogeneity of base composition across species for total, intron, exon, and three codon sites of the concatenated data set. The results indicated that four nucleotide bases occurred in almost equal proportions and the GC content varied little among species for all data partitions ( $\chi^{2}$ tests, $P=0.346-1.0$; Additional data file 3 ). The 
Table I

\begin{tabular}{|c|c|c|c|c|c|}
\hline Species & Genome & Accession number* & Origin & $\begin{array}{l}\text { No. of genes } \\
\text { sequenced }\end{array}$ & No. of sites aligned \\
\hline Oryza sativa† & A & $93-11$ & China & 62 & 52,092 \\
\hline O. rufipogon & A & 105480 & India & 142 & 124,079 \\
\hline O. barthiit & A & 104132 & Cameroon & 62 & 52,092 \\
\hline O. punctata & B & 103903 & Tanzania & $14 \mid$ & 124,079 \\
\hline O. officinalis & C & 104972 & China & 142 & 124,079 \\
\hline O. rhizomatis $\dagger$ & C & 103410 & Sri Lanka & 62 & 52,092 \\
\hline O. eichingeri† & C & 105415 & Sri Lanka & 62 & 52,092 \\
\hline O. australiensis & $E$ & 105263,101410 & Australia & 135 & 124,079 \\
\hline O. brachyantha & $\mathrm{F}$ & 105151 & Sierra Leone & 124 & 124,079 \\
\hline O. granulata & G & M8-15, 106469 & China, Vietnam & 124 & 124,079 \\
\hline Leersia tisserantti & - & 105610 & Cameroon & 122 & 124,079 \\
\hline
\end{tabular}

*All accession numbers were obtained from the International Rice Research Institute at Los Banos, Philippines, except for M8- I5, which was collected by the authors. SSixty-two genes were sequenced for these species and used only for testing the effect of dense sampling. Sequences of $O$. sativa $(93-I I)$ were retrieved from the BGI-RIS database.

potential compositional bias was also examined with analysis using log-determinant (LogDet) distance [36]. This yielded the same topology as ML, MP, and BI (Table 2). These tests suggest that the concatenated data set did not contain compo-

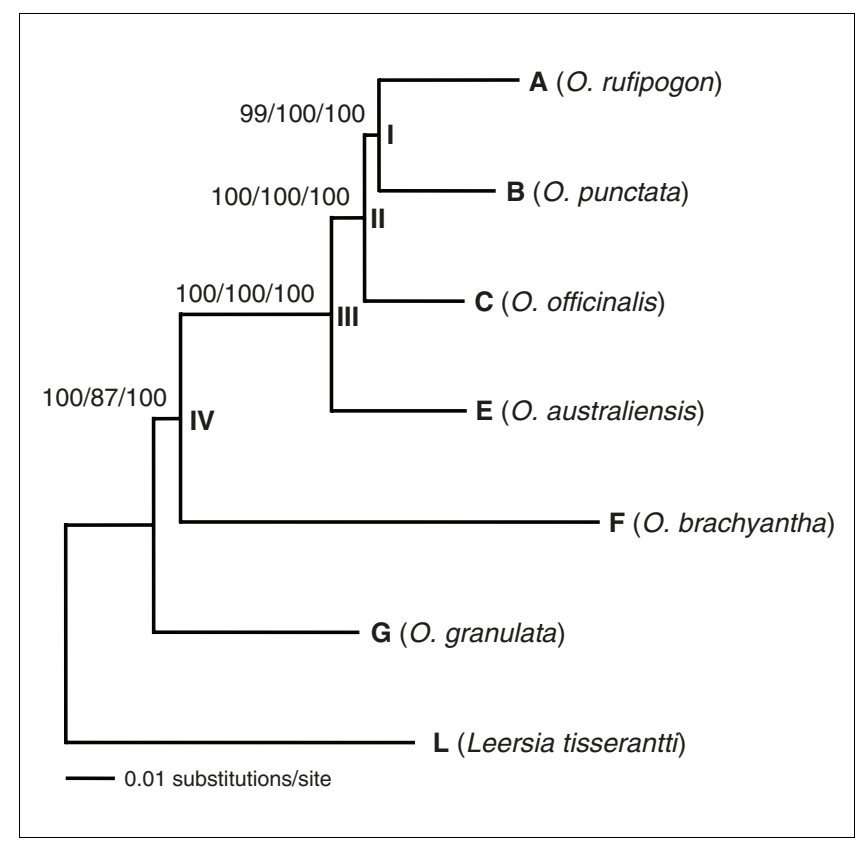

\section{Figure I}

$M L$ tree inferred from the concatenated sequences of 142 genes using the $\mathrm{GTR}+\Gamma$ model. The same topology was obtained from MP and $B I$. The letters $\mathrm{A}, \mathrm{B}, \mathrm{C}, \mathrm{E}, \mathrm{F}$, and $\mathrm{G}$ represent all recognized diploid genome types of Oryza, and $L$ represents the outgroup. The names of the species that represent the genome types and outgroup are in parentheses. Numbers above branches indicate bootstrap support of $M L$ and $M P$, and posterior probability of $\mathrm{BI}$, respectively. Four internal branches of Oryza genome types are indicated with I, II, III, and IV. Branch length is proportional to the number of substitutions measured by the scale bar. sitional signals that could have biased the phylogenetic reconstruction.

Second, we analyzed rate constancy among lineages using Tajima's relative rate test [37]. When the concatenated sequences were considered, results showed that the null hypothesis of rate constancy was rejected in almost all pairs of contrasts $(P<0.01)$. It is noteworthy that the F-genome evolved at a faster rate and the G-genome evolved at a slower rate than other genomes (Additional data file 4). To explore the potential impact of rate heterogeneity on tree reconstruction, we adopted the RY-coding strategy that discards fastevolving transitions and consequently makes phylogenetic reconstructions less susceptible to uneven occurrence of multiple hits among lineages [34,38]. The tree obtained from the re-coded data set was topologically identical to that shown in Figure 1 (Table 2). To further test the potential long-branch attraction effect of the fast-evolving F-genome, we identified genes that evolved more rapidly in the F-genome than in the A-, B-, and C-genomes. We calculated the ratio of the mean distance between the F-genome and each of A-, B-, and Cgenomes to the mean distances among A-, B-, and C-genomes for each gene. We then progressively excluded fast-evolving genes of the F-genome in a decreasing order of the ratios. The topology based on the remaining genes did not change until more than 50 genes were excluded (Additional data file 5). These suggest that rate heterogeneity was not severe enough to cause significant systematic bias.

Third, to examine the potential systematic errors caused by model misspecification [30,32,35], we applied a series of homogeneous and mixed models in BI and evaluated the relative merits of competing models by Bayes factors. Although Bayes factor comparisons showed that all mixed models outperformed the homogeneous models significantly (Additional 
Table 2

\begin{tabular}{|c|c|c|c|c|}
\hline \multirow[b]{2}{*}{ Method } & \multicolumn{4}{|c|}{ Bootstrap support (\%) } \\
\hline & I & II & III & IV \\
\hline RY-coding strategy (ML) & 93 & 100 & 100 & 73 \\
\hline LogDet distance $(\mathrm{N} J)$ & 93 & 100 & 100 & 90 \\
\hline Gene bootstrap (ML) & 72 & 100 & 100 & 88 \\
\hline
\end{tabular}

$\mathrm{NJ}$, neighbor-joining.

data files 6 and 7), analyses with all 14 alternative models, including ones incorporating the covarion model, which accounts for heterotachous signal, yielded the same topology as shown in Figure 1, with all internal branches supported by 100\% PP. Taken together, the above analyses indicate that the phylogeny inferred from the concatenated gene sequences was not biased by systematic errors.

We next tested whether the resulting phylogeny could have been influenced by a subset of the genes. A gene bootstrap analysis was performed with 1,00o replicates [27]. For each replicate, we randomly drew 142 genes with replacement from the entire pool. The sampled genes were concatenated and analyzed using ML. The results strongly supported the topology shown in Figure 1 (Table 2), indicating that the phy-

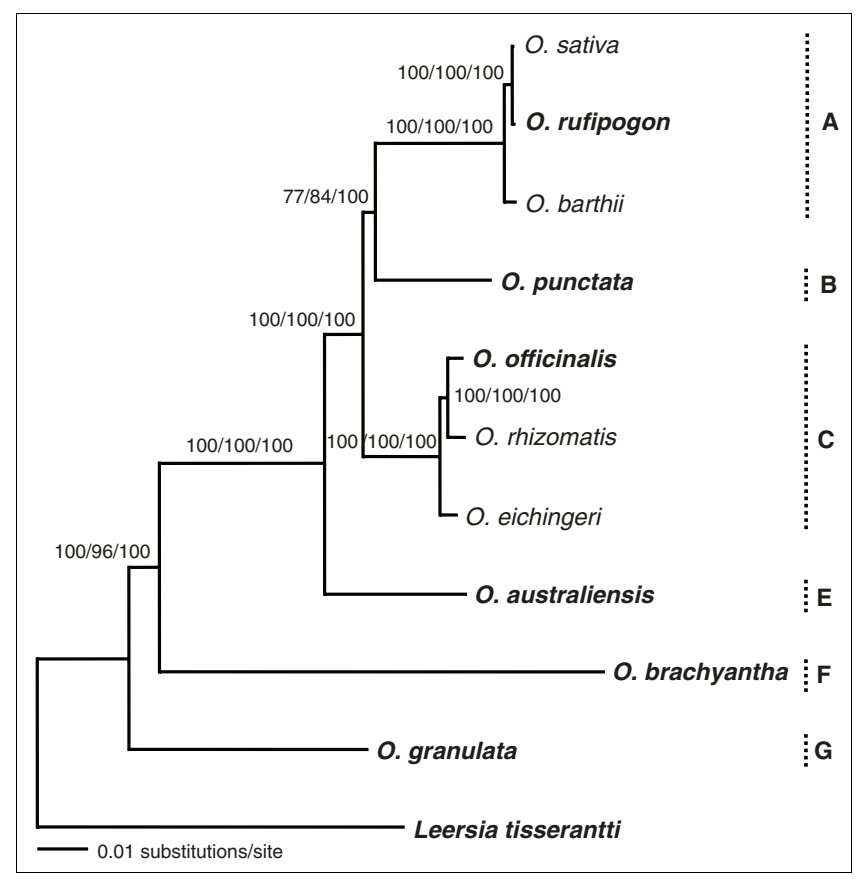

Figure 2

$M L$ tree inferred based on concatenation of 62 genes from II species using the $G T R+\Gamma$ model. Numbers above branches indicate bootstrap support of ML and MP, and posterior probability of $B I$ analyses, respectively. Capital letters (A to $G$ ) beside the tree specify the genome type of the species. For the species in bold, 142 genes were sequenced and used in the analyses as shown in Figure I. logenetic reconstruction was not dominated by a subset of the 142 genes.

Finally, we investigated whether within-genome sampling would influence the phylogenetic reconstruction. Because the A- and C-genomes have more than one species, while each of the remaining genomes has only one species $[1,9,13]$, we sequenced 62 genes for an additional A-genome species, $O$. barthii, and two additional C-genome species, O. rhizomatis and $O$. eichingeri (Table 1). Sequences of cultivated rice, $O$. sativa, belonging to the A-genome were also retrieved from the BGI-RIS Database [39] and added to the data set. Phylogenetic analyses of the 11 species generated the same intergenome relationship as the one shown in Figure 1 (Figure 2). This indicates that one species sampled from each genome was sufficiently representative for the reconstruction of the genome relationships.

\section{Phylogenetic incongruence and network analyses}

When phylogenetic analyses were done for each of the 142 genes separately, more than 40 different optimal trees were generated, indicative of extensive incongruence among gene phylogenies. To gain insight into the extent of incongruence, we constructed consensus networks from ML trees of the 106gene data set without missing data. Figure 3 a shows the network at a threshold of 0.15 , which presents branches appearing at a frequency of $15 \%$ or higher of all gene trees. Two boxes are evident in the network, indicating that topological incongruence is concentrated on branch I involving the relationships between A-, B-, and C-genomes and branch IV involving the relationships between the F-genome, G-genome, and the rest of the genome types (R). We also explored the features of consensus networks by increasing the threshold from 0.05 and found that the boxes were collapsed when the threshold reached 0.3 and it ended up with the topology identical to that shown in Figure 1 (Additional data file 8). These results further support the phylogenetic relationships revealed by the concatenated data set and highlight the incongruence involving branches I and IV.

In the first box, the length of parallel edges supporting split $\mathrm{AB} \mid \mathrm{CEFGL}$ is longer than those supporting splits $\mathrm{AC} \mid \mathrm{BEFGL}$ and $\mathrm{BC} \mid \mathrm{AEFGL}$ (Figure 3a), suggesting that a higher proportion of consensus signal groups A and B together. This is in 
(a)

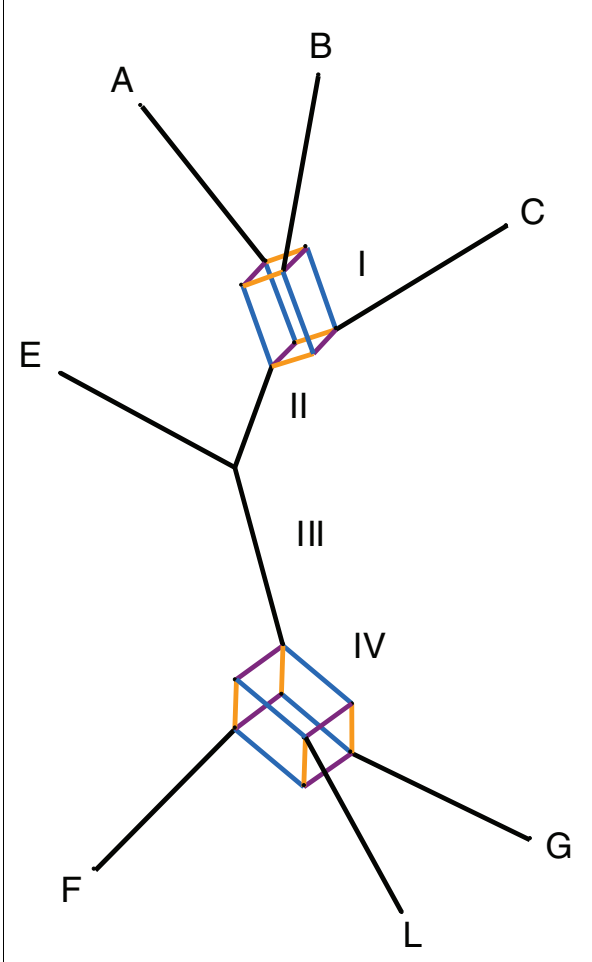

(b)
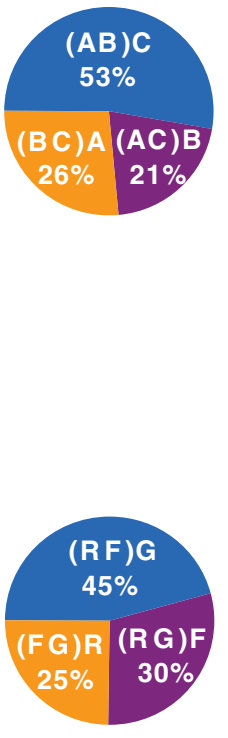

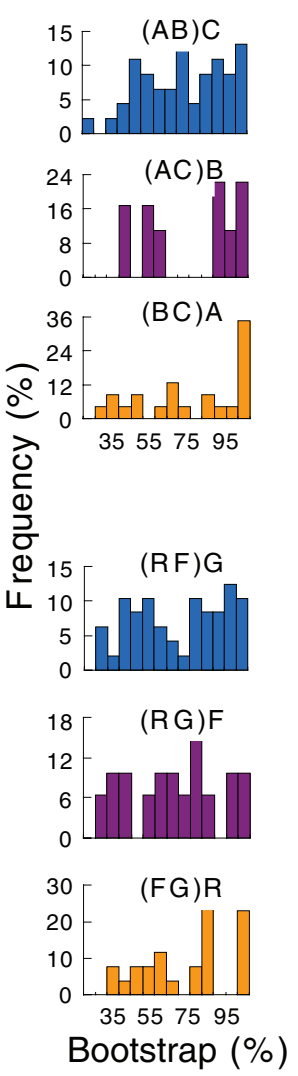

(c)

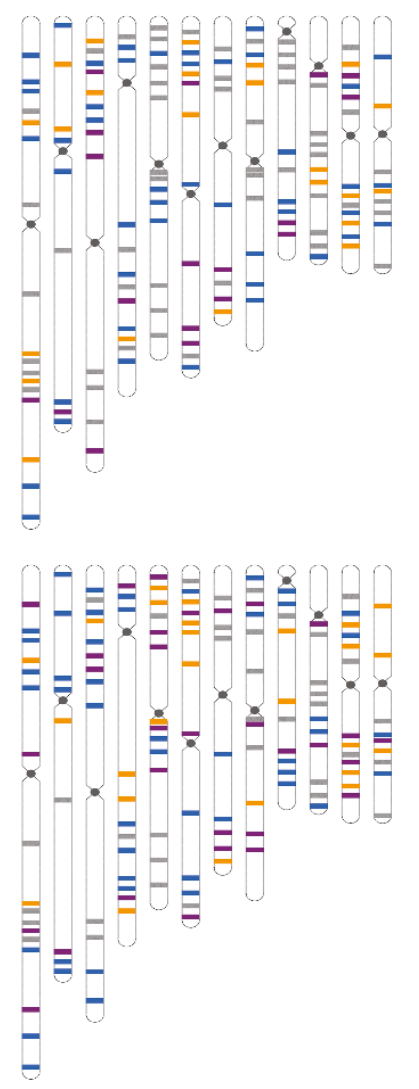

Figure 3

Genome-wide incongruence. A, B, C, E, F, and G represent Oryza genome types and L represents the outgroup, Leersia. (a) Consensus network constructed from ML trees at a threshold of 0.15 . The two boxes indicate the relatively high levels of incongruence among gene trees associated with internal branches I and IV. Branch length is proportional to the frequency of occurrence of a particular split of all gene trees. R represents the rest of the genome types, including A-, B-, C-, and E-genomes. Color schemes: for the box associated with branch I, blue, orange, and purple illustrate splits supporting alternative topologies, $(A B) C,(B C) A$, and $(A C) B$, respectively; for the box associated with branch IV, blue, orange, and purple illustrate splits supporting alternative topologies, (RF)G, (FG)R, and (RG)F, respectively. (b) Pie graphs indicate the proportions of gene trees that support alternative splits in the corresponding boxes at the left. Histograms at the right illustrate the distribution of ML bootstrap support for the corresponding split (in the corresponding colors). (c) Illustration of the relative physical locations of the 142 sampled genes on the 12 rice chromosomes based on rice genome sequences. The colors indicate genes supporting a split or topology coded in the same color in the corresponding boxes on the consensus network. Genes coded in gray are those that had no input in the topology illustrated in the pie graphs and those not included for the construction of the consensus network because of missing data.

agreement with the result that a larger number of gene trees (53\%) support the sister relationship of A and B than those supporting the alternative sister relationship between $\mathrm{B}$ and C (26\%) or between A and C (21\%) (Figure 3b). For the second box, the length of parallel edges supporting split ABCEF|GL is longer than those supporting the two alternative splits. This is also consistent with the result that $45 \%$ of gene trees support the sister relationship between $\mathrm{R}$ and $\mathrm{F}$ while $30 \%$ and $25 \%$ of gene trees support the sister relationships between $\mathrm{R}$ and $\mathrm{G}$ or between $\mathrm{F}$ and $\mathrm{G}$, respectively (Figure $3 \mathrm{~b}$ ).

To further explore the incongruence among gene trees, we performed the incongruence length difference (ILD) test based on two partitioning strategies and found that there was no significant incongruence between any pair of the process partitions (intron and three codon positions; Additional data file 9). In contrast, significant heterogeneity was found among gene partitions, including tests among all gene partitions as a whole $(P<0.01)$ and between pairwise comparisons and between each gene and the remaining genes combined (Additional data file 10). These results were consistent with the distributions of bootstrap support for alternative topologies at the two boxes (Figure $3 \mathrm{~b}$ ). For each box, there is a substantial proportion of high bootstrap support for alternative topologies, suggesting that the competing topologies are well supported on the respective gene trees. Remarkably, genes supporting any given topology are distributed randomly 
among the 12 chromosomes ( $\chi^{2}$ test, $P=0.233-0.823$ ), indicative of a genome-wide incongruence (Figure $3 \mathrm{c}$ ).

To address the question of whether the incongruence among genes is attributed to different evolutionary histories of genes or merely systematic errors [40,41], we conducted tests for systematic bias for each of the 142 genes. The Chi-square test revealed that there was no heterogeneity of base composition for any gene. However, rate heterogeneity was detected for some genes by the relative rate test. We then conducted phylogenetic analyses for each gene using different strategies, including ML, MP, RY-coding, and LogDet distance. The comparison of bootstrap 75\% majority-rule consensus trees showed that only 4 out of 142 genes yielded incompatible topologies between different methods of analyses (Additional data file 11). This indicates that there are few systematic errors involved in individual genes and the incongruence among gene partitions is governed mainly by different evolutionary histories of genes.

\section{Short branches and their resolution}

Different evolutionary histories of genes can be attributed to three major factors, including paralogy, hybridization, and lineage sorting [40]. We have largely ruled out the potential effect of paralogy by carefully screening gene markers (see Materials and methods). The pattern of incongruence also does not support hybrid speciation because hybridization would have led to two major incongruent topologies rather than the presence of a leading topology with two alternative topologies occurring at nearly equal frequencies for both clades I and IV. The random distribution on chromosomes of the genes that support a given topology (Figure 3c) does not support the hybridization hypothesis either because related or linked loci should share gene trees if the species have a history of introgression or hybridization [42]. Therefore, we are left with the hypothesis of lineage sorting as the primary explanation for the incongruence.

Population genetic theory suggests that lineage sorting is more likely to occur at an internal branch of a species tree that is short (few in generations) and wide (large in effective population sizes) [43,44]. Based on estimation by the ML method, branches I and IV were the shortest internal braches on the concatenation tree and obtained relatively low support values in analyses with different methodologies (Figure 1 and Table 2). For branch I, there is a sufficient amount of published data that allow us to estimate the probability to obtain the species tree from a given gene. That is, $P=1-2 / 3 \exp (-t)$ under the coalescent model, where $t$ is the time between two speciation events in the unit of generations/2Ne and $\mathrm{Ne}$ is the effective population size [43].

Using the previously reported nucleotide diversity at silent sites $\left(\theta_{\text {sil }}=0.0038-0.0095\right)$ for the A- and C-genome species $[45,46]$ and a substitution rate for grasses $\left(5.9 \times 10^{-9}\right.$ substitutions per synonymous site per year) $[47,48]$, we estimated that the effective population sizes of these Oryza species ranged from $1.6 \times 105$ to $4.0 \times 105$. A speciation model test on three Cgenome species suggested that their ancestral population sizes were approximately ten-fold larger than those of each species [45]. Thus, the ancestral population size of A-, B-, and Cgenomes $(\mathrm{Ne})$ should be at least $1.6 \times 10^{6}$. Because the Agenome species began to diverge approximately 2 Mya [49] and divergence between B- and C-genomes occurred approximately 3.8 Mya [45], the time between two speciation events should be less than 1.8 million years. Given the generation time of 1-2 years in wild rice species [50], the number of generations between the two speciation events is at most $1.8 \times$ $10^{6}$. The estimated upper limit of generations together with the lower limit of $\mathrm{Ne}$ led to the calculation of the upper limit of $P$ as 0.62 . This implies that there is less than a $62 \%$ chance for any given gene tree to be the same as the species tree or less than $62 \%$ of gene trees from the sampled genes will be congruent with the species tree. Our finding that $53 \%$ of gene trees support the sister relationship of A- and B-genomes agrees with the theoretical expectation (Figure $3 \mathrm{~b}$ ), which further supports the lineage sorting hypothesis.

For branch IV, the divergence happened at greater depth in the tree and thus homoplasy resulting from mutational saturation might be a factor to cause incongruent gene phylogenies [33,51]. However, analyses of saturation plots did not reveal any mutational saturation for the concatenated data set (Additional data file 12), suggesting that lineage sorting is still the most plausible explanation for the incongruence.

To assess how much of the data set might be needed to resolve such short branches, we explored the relationship between the number of genes or nucleotide sites and the proportion of gene trees that support the topology or clades shown in Figure 1. The results demonstrated that the probability of getting identical topology or clades as in Figure 1 steadily increased with the number of genes or sites sampled, regardless of methods used, although ML generally performed better than MP (Figure 4). Using 95\% of identical gene trees or clades in 500 replicates as a criterion, about 120 genes were needed for both ML and MP methods to resolve branch I and more than 80 (ML) and 120 (MP) genes were needed to resolve branch IV. Additionally, 120 (ML) or more genes (MP) were needed to resolve both branches simultaneously (Figure 4 and Additional data file 13).

When nucleotide sites rather than genes were the unit of resampling, about $40 \mathrm{~kb}$ of nucleotides were sufficient to resolve branch I with both methods. This is equivalent to 46 sampled genes in length given the average length of 874 bp per gene. It took approximately $40 \mathrm{~kb}$ and $80 \mathrm{~kb}$ (approximately 92 genes) for ML and MP, respectively, to resolve branch IV. A total of $50 \mathrm{~kb}$ (approximately 57 genes) for ML and $80 \mathrm{~kb}$ for MP were sufficient to resolve both branches simultaneously (Figure 4 and Additional data file 13). These results indicate that random sampling of unlinked nucleotides has a higher 


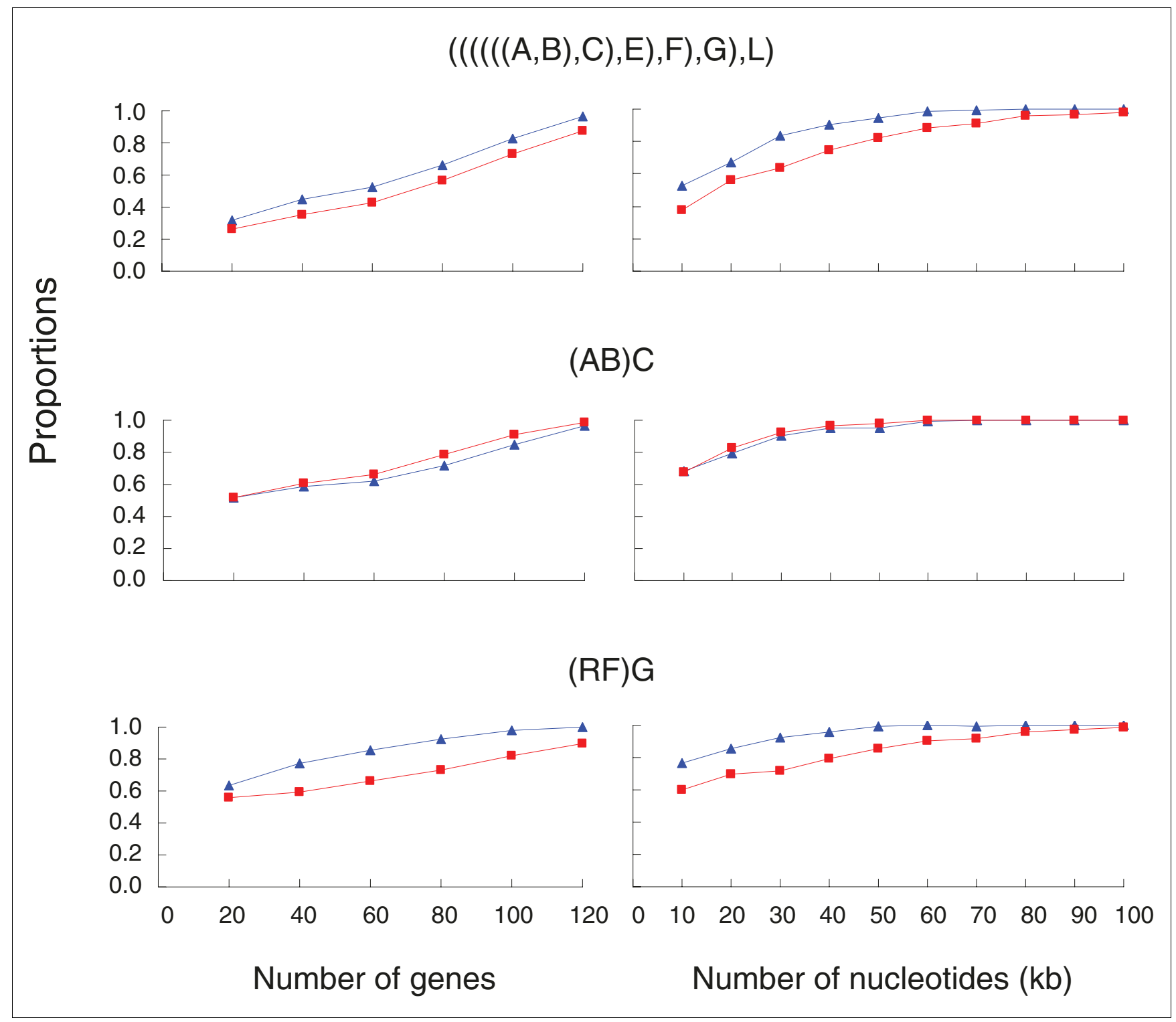

Figure 4

The proportions of topologies (or clades) that are identical to those shown in Figure I based on resampling of I42 gene sequences at various scales. Results of ML and MP analyses are indicated by blue and red, respectively. Genome types are represented with the same capital letters as in Figure 3.

power of phylogenetic resolution than sampling contiguous nucleotides such as those within a gene.

\section{Discussion}

This study fully resolved the phylogeny of the rice genomes. Through extensive tests and analyses, we demonstrate that the phylogenetic reconstruction based on the sequences of 142 genes was not biased by systematic or sampling errors and was insensitive to phylogenetic methods or model specification. We identified across the genome a remarkable level of incongruence of gene phylogenies at the two shortest internal branches (Figures 1 and 3). Our analyses clearly indicated that lineage sorting was a primary cause for the difficulty of resolving two branches of the rice phylogeny that underwent rapid diversification. Even more remarkably, lineage sorting occurred for genes distributed randomly across all 12 rice chromosomes (Figure $3 \mathrm{c}$ ). This study thus documents a case of genome-wide lineage sorting that gave rise to species with the mosaic of ancestral genomes [26,52]. One implication of our findings is that special caution must be taken in interpreting phylogenetic relationships of rapidly diverged lineages even though the relationships are strongly supported on a single gene phylogeny. Our results also imply that although it may not be feasible to have a large number of genes to resolve a short branch for groups with limited genomic resources, utilization of a few genes should provide a clue to the extent to which lineage sorting may lead to erroneous phylogenies [12]. 
The biological implications for the presence of two short branches (I and IV) that reflect two episodes of rapid diversification of the rice genus are profound. Based on a molecular clock estimate, the first event occurred approximately 10 Mya [53] and led to a rapid diversification of the G-genome, Fgenome and a lineage that subsequently diversified into the rest of the rice genomes. Additionally, the $\mathrm{H}-, \mathrm{J}-$, and $\mathrm{K}-$ genomes that are now only present in extant tetraploid species, including $O$. longiglumis and $O$. ridleyi with the HJgenome and $O$. schlechteri and $O$. coarctata with the HKgenome, also diverged around this time $[12,53]$. The second event led to the diversification of A-, B-, and C-genomes approximately 5 Mya [45,53]. Therefore, the two episodes of rapid diversification gave rise to almost the entire diversity of the genus. Because the Oryza species are distributed in distinct habitats across four continents $[1,50]$, it would be interesting to further investigate whether the rapid diversification was coupled with adaptive radiation under certain geological and ecological conditions $[54,55]$.

Rapid speciation, particularly ancient radiation, featured by the short internal branches in phylogenetic trees, poses an extraordinary challenge to systematic and evolutionary biologists $[33,51,55,56]$. It has been observed at a variety of time depths ranging from as early as the Cambrian explosion of animal phyla over 550 Mya [25] to as recent as the divergence between human, chimpanzee, and gorilla a few Mya $[52,57,58]$. In many cases, phylogenetic relationships seemed to be an irresolvable polytomy $[23,56,59]$ because of the rapid radiations. Such closely spaced series of speciation events was accordingly considered to be "bushes in the Tree of Life" [33]. To date, rapid evolutionary radiations have been proposed to be the most plausible explanation for the poorly resolved phylogenies or polytomies in many organisms such as aphids, black flies, bees, birds, turtles, mammals, and higher plants $[29,30,33,51,60]$. However, a growing body of evidence showed that many assumed polytomies were 'soft' and could be resolved into sequential bifurcations with additional data and proper methods of phylogenetic analysis [52,59,61,62]. In a study of phylogenetic relationships among tetrapod, coelacanth, and lungfish, Takezaki et al. [59] obtained an irresolvable trichotomy although sequences of 44 nuclear genes were analyzed. Using computer simulation, they concluded that more than 200 loci would have to be analyzed to resolve the relationships among the three lineages if the fish-to-tetrapod transition interval was 10-20 million years long. The once unresolved relationship among human, chimpanzee, and gorilla is a typical example of soft polytomies. Recent analyses with an increased amount of molecular data resolved human and chimpanzee into a sister group [52,57,58]. Our results exemplify that rapid speciation within an angiosperm genus can be reliably resolved as long as a sufficient amount of unlinked DNA sequences is available.

However, we should also realize that the increase in the amount of data alone may not provide a universal solution to all short branches on the Tree of Life. It is theoretically possible that certain branches are not resolvable even with whole genome sequences if time intervals between speciation were extremely short and the speciation events were sufficiently ancient $[31,33,51]$. These branches are considered to be 'hard' polytomies [33,61]. Nevertheless, both soft and hard polytomies provide historical information on evolutionary processes and a phylogenetic analysis with genome-wide information can be most helpful for understanding the evolutionary histories behind these seemingly problematic, but perhaps intriguing, branches of the Tree of Life.

For soft polytomies, an obviously interesting question is how many DNA sequences would be needed to resolve rapid speciation considering that DNA sequences have been, and will remain, major sources of biological data $[31,32]$. The mosaic genome or different evolutionary histories of genes under rapid speciation, in conjunction with other factors associated with species divergence (for example, selection and high homoplasy of ancient speciation [33,51]), brings about difficulties in resolving speciation events when using a small number of regions/genes or limited characters [22,59]. This study shows that as many as 120 genes with an average length of $874 \mathrm{bp}$ or $50 \mathrm{~kb}$ of randomly sampled nucleotides from 142 genes are needed to resolve clades I and IV simultaneously with over 95\% confidence (Figure 4). Clearly, blocks of contiguous nucleotide sites were less powerful in phylogenetic resolution than samples consisting of sites drawn randomly from the genome because nucleotides within genes do not evolve independently $[22,63]$. This implies that for the same amount of sequence data, a larger number of unlinked shorter DNA fragments are preferred over a smaller number of larger fragments for resolving short branches.

\section{Conclusion}

As the speed of genome sequencing continues to accelerate, phylogenomics is becoming a growing field of evolutionary biology. The potential of phylogenomics to address fundamental evolutionary questions has yet to be realized with the accumulation of phylogenomic studies for diverse groups of organisms [31-33]. The successful resolution of the rice phylogeny demonstrates the power of phylogenomics in the reconstruction of rapid evolutionary diversification. This study also highlights that organismal genomes might be mosaics of conflicting genealogies because of rapid speciation and exemplifies that phylogenetic relationships of organisms that undergo explosive or rapid diversification can be reliably resolved with increasing amounts of data and improved analytical methodology. A fully resolved rice phylogeny lays a solid foundation for comparative and functional genomic studies of rice and its related species and genera. Combined with the availability of rice genome sequences $[2,64]$ and the BAC libraries of Oryza species representing all rice genome types [7], this phylogenetic framework will play an important 
role in the studies of genome evolution, speciation and adaptation, and crop domestication.

\section{Materials and methods Sampling single-copy genes}

We used the BGI-RIS Database [39] for gene screening. Similar to the strategy used by Yu et al. [64], we extracted the protein sequences with nr-KOME cDNA [65] evidence and then conducted extensive searches against the genomic sequences of indica rice (93-11) in all six reading frames using TBLASTN at E-values of $10^{-7}$. To ensure that single-copy genes were used in our analysis, we applied a stringent similarity criterion of $50 \%$ in our searches; that is, only protein-coding genes that have no counterpart over 50\% similar to themselves in the rice genome were selected for further analyses. Excluding those sequences without syntenic counterparts in the japonica (Nipponbare) genome [2], we got a total of 943 genes as candidates for phylogenetic markers. Using coding sequences of these candidates, we performed BLAST searches against the GenBank database to obtain the gene counterparts from barley, maize, sorghum, wheat, or other species of Poaceae as targets for primer design. On this basis, we designed 162 pairs of primers for amplifying orthologous segments from Oryza species and the outgroup Leersia tisserantti. Finally, 118 genes were kept according to the following criteria: they were sampled randomly from all the 12 rice chromosomes; the amplifying length ranged from 0.5-2.o kb with an intron length of $30-70 \%$ so that adequate information is available at different taxonomic levels; and clear and strong amplified fragments were obtained from the Oryza species and the outgroup. Moreover, we sequenced 24 additional genes that were single copies demonstrated by previous studies (Additional data file 2). All the 142 genes used in this study were mapped onto the chromosomes of indica rice (93-11) (Additional data file 1).

\section{Species sampling, amplification, and sequencing}

We sampled six Oryza species, representing all six diploid genomes in the genus, and one Leersia species (L. tisserantti) as outgroup because Leersia is most closely related to Oryza $[12,53]$. Information on the materials used in this study is listed in Table 1. Primers for PCR of all 142 genes are listed in Additional data file 14. Missing or partial sequences of some genes were present in some species because of the amplifying difficulty (Table 1). However, missing data in our case did not impact the tree constructions no matter what methods were used because our data set contained sufficient information, consistent with previous computer simulation and empirical investigation $[21,25,66]$.

PCR amplifications and purification of the products were performed by standard methods. Purified products were sequenced either directly or after cloning into pGEM T-easy vectors (Promega, Madison, WI, USA) if the direct sequencing failed. Sequencing was carried out on an ABI 3730 auto- mated sequencer (Applied Biosystems, Foster City, CA, USA). All sequences obtained in this study have been deposited in the GenBank database (accession numbers EF577518 to

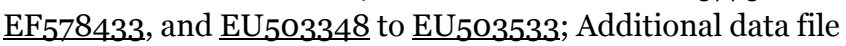
14).

\section{Phylogenetic reconstructions}

Individual genes were aligned using T-Coffee [67] and then manually adjusted. Phylogenetic trees were reconstructed by ML, MP and BI methods. ML and MP were implemented with PAUP 4.ob10 [68] and the branch-and-bound algorithm was used for tree searching. A non-parametric bootstrap strategy [69] was used for assessing tree reliability, with 1,00o replicates for MP analysis and with 100 and 500 replicates for ML analysis of the concatenated sequence and single genes, respectively.

BI was attempted with MrBayes 3.1.2 [70]. Given the sensitivity of the Bayesian method to model misspecification, we explored a series of homogeneous models by combining model components in different ways, including substitution rates among nucleotides (Nst $=1,2,6)$, rate variations across sites (Rates = Equal, Gamma, Propinv, Invgamma), and rate variations across the tree (Covarion $=$ Yes, No) (Additional data file 6). Furthermore, we explored mixed models that accommodate heterogeneity across data partitions by specifying partition-specific substitution models [70]. We applied mixed models to our partitioned data by two schemes (see 'Analysis of systematic bias and congruence tests' below). Mixed models were implemented with separate models for each data partition selected by the program Modeltest 3.7 [71] and model parameters separately estimated, and a rate multiplier (ratepr $=$ variable) was also employed to allow the overall rate to be different across partitions. In all the BI analyses, three independent Markov Chain Monte Carlo runs were executed, each starting with randomly choosing topologies for the four simultaneous chains, one cold and three incrementally heated. The four chains were run for at least 1,000,000 generations until stationarity in Markov chains was achieved, sampling trees every 100 generations with the first $10 \%$ of trees sampled discarded as burn-in, and then the posterior probabilities were calculated from the remaining samples.

We used Bayes factors [72] to evaluate the relative merits of two competing models, with the intention of detecting the effect of model components on our data. This method does not require alternative models to be hierarchically nested, and so it makes possible the comparison of any pair of distinctly different models. A Bayes factor in favor of one model (model 1) over another model (model o) was calculated as the ratio of their marginal likelihoods and the natural logarithm of marginal likelihood can be approximated by the harmonic mean of the likelihoods of Markov Chain Monte Carlo samples with MrBayes [73]. We calculated twice the natural logarithm of the Bayes factors for the competing model pairs, and 
interpreted the results according to the rule suggested by Kass and Ratery [72], which states that a result of 2 to 6 is 'positive' evidence in favor of model 1, a result of 6 to 10 is 'strong' evidence, and a result of $>10$ is 'very strong' evidence; conversely, a result of $<$ o provides evidence in favor of model o.

\section{Phylogenetic network analysis}

To combine evidence from different loci without losing the information on independent gene histories, which might be drowned out by suppressing them into a bifurcating tree, several phylogenetic network approaches have been proposed and proven to be useful alternatives when using multi-gene data sets [74-76]. Consensus network, which is applied to multiple trees with the same set of taxa, is one commonly used network approach and can display simultaneously the conflicting evolutionary hypotheses based on multiple loci in a network fashion $[74,76]$. Such conflict or uncertainty might arise from stochastic errors, systematic bias, or biological processes [75]. Therefore, phylogenetic networks provide a more inclusive approach than analysis of the concatenated data set because weak or conflicting signals are hidden when genes are concatenated before phylogenetic analysis [76].

In the consensus network, areas where all trees have compatible splits (that is, a split is a bipartition of the taxa) will be tree-like (that is, a single branch); in contrast, areas with incompatible splits will be represented by bands of parallel edges, thus forming a potentially hyper-dimensional graph. The degree of denseness of boxes in networks reflects the intensity of contradictory evidence for grouping certain taxa, and the length of an edge is determined by the weight assigned to it $[74,75]$. The phylogenetic networks can range from one extreme, a structure of high-dimensional hypercubes in the absence of any common phylogenetic patterns among gene trees, to the other extreme, a unique bifurcating tree in the absence of stochasticity associated with bifurcating evolutionary process [75]. By employing the threshold value, we can reduce the visual complexity of resulting graphs by using only the splits that occur in more than a given proportion of all trees.

In the present study, we constructed consensus networks from optimal ML trees for a 106-gene data set in which sequences of all six diploid genomes and the outgroup were available and included in our consensus network all splits that occurred above a threshold value ranging from 0.05-0.3. In our case, branch lengths were not considered when using optimal ML trees as source trees because we were only interested in the conflict between topologies of gene trees. Thus, edge lengths in the final network are proportional to the number of trees in which a particular split appears. Consensus network was performed by the method described by Holland [76], in which Python scripts (kindly offered by BR Holland) was first implemented to create Nexus files and then the resulting network was visualized by Spectronet [77].

\section{Analysis of systematic bias and congruence tests}

Systematic errors such as compositional signal, rate signal and heterotachous signal might be reinforced as more and more data are considered [35]. We first tested the compositional bias resulting from the heterogeneity of nucleotide compositions among lineages by Chi-square test. The LogDet distance [36] was also used to account for compositional bias with the neighbor-joining method. Then Tajima's relative rate test [37] was employed with each pair of Oryza species, using $L$. tisserantti as outgroup, to test rate constancy. Sequence data were also analyzed under the RY-coding strategy (A and $\mathrm{G}=\mathrm{R}, \mathrm{C}$ and $\mathrm{T}=\mathrm{Y}$ ), which maintains only transversions and thus efficiently reduces saturations by excluding more frequently occurring transitions [31,38]. In addition, the effect of heterotachous signal was explored by implementing a covarion model in BI.

Substitutional saturation of the data set was evaluated by plotting observed pairwise distance (uncorrected P-distance) for transitions and transversions against the ML pairwise distances for each pair of taxa. Saturation plots were constructed for total, exon, intron and third codon positions, respectively. Second order polynomial regression lines were fitted to all saturation plots and if the slope of this regression line was zero or negative, the data were considered saturated [78].

The ILD test [79], a character-based test for homogeneity, was used to explore the difference in phylogenetic signal between data partitions. We partitioned the data set by two schemes: four process partitions including intron and each codon positions [8o]; and 142 gene partitions along gene boundaries, which may reveal variation in allelic histories that the concatenated data might obscure $[26,76]$. Then, we performed three kinds of ILD tests for each type of partition: a test among all partitions simultaneously; a test between all possible pairwise partitions; and a test between single partitions and the rest of the data set combined.

\section{Amount of sequence and phylogenetic resolution}

To explore the relationship between the number of genes or nucleotides in a sample and the probability to infer the species tree in our case, we drew random samples of different sizes from the original 142-gene data set without replacement and concatenated each sample for phylogenetic analyses. When sampling genes, we generated samples consisting of $20,40,60, \ldots, 120$ genes each for 500 replicates. Similarly, samples with randomly sampled sites in a total length of 10 , $20,30, \ldots 100 \mathrm{~kb}$ were generated each for 500 replicates. ML and MP methods were used to determine whether or not the sampling results were affected by reconstruction methods. The branch-and-bound search was used in both methods, with the General Time Reversible (GTR) $+\Gamma$ model for ML. The proportion of trees (or clades) identical to that in Figure 1 was calculated as the probability that a correct phylogenetic hypothesis will be obtained at a specific data size [63]. 


\section{Abbreviations}

BI, Bayesian inference; GTR, General Time Reversible; ILD, incongruence length difference; $\mathrm{kb}$, kilo base pairs; LogDet, log-determinant; ML, maximum likelihood; MP, maximum parsimony; Mya, million years ago; PP, Bayesian posterior probability.

\section{Additional data files}

The following additional data are available with the online version of this paper. Additional data file 1 is a figure showing the relative location on rice chromosomes of the 142 genes sampled in this study. Additional file 2 is a table listing the detailed information on each of 142 loci. Additional file 3 is a table listing the GC content variation among lineages and the result of Chi-square test for the concatenated data set. Additional file 4 is a table summarizing the Tajima's relative rate test for concatenated sequences using Leersia as outgroup, with estimates of the ratio of substitution rate between lineages. Additional file 5 includes figures showing the results of testing the effect of rate bias caused by the fast-evolving genes of the F-genome. Additional file 6 is a table summarizing 14 alternative models used in BI analyses. Additional file 7 is a table indicating the effect of model components on model fit judged by Bayes factor comparisons of competing models. Additional file 8 is a figure showing the consensus networks of a collection of 106 optimal ML trees from the 106 genes with the complete set of seven species, applying thresholds of $0.05,0.1,0.15,0.2,0.25$ and 0.3 , respectively. Additional file 9 is a table presenting the results of the ILD test for pairwise comparisons of process partitions. Additional file 10 is a table summarizing the number of genes that failed the ILD test with the target gene at $P<0.01$ for total, intron, exon and the third codon sites, respectively, and the $P$ value of the ILD test between the target gene and all the rest of genes. Additional file 11 is a table presenting the topologies of bootstrap 75\% majority-rule consensus trees by different methods of analyses for each gene. Additional file 12 is a figure showing saturation analyses in the concatenated datasets of total, intron, exon, and third codon positions, respectively. Additional file 13 is a table summarizing the proportions of topology (or clades) identical to those shown in Figure 1 inferred from randomly sampled genes or sites in 500 replicates. Additional file 14 is a table listing the primers for PCR amplification and the GenBank accession numbers of the sequences of 142 loci sampled in this present study.

\section{Authors' contributions}

SG, XHZ, and TS designed the study; XHZ, FMZ, LLZ, and SG performed the research; FMZ, JGZ, and JW contributed the gene screening; XHZ, FMZ, LT, SG, and TS analyzed the data; SG, TS, and XHZ interpreted the data and wrote the paper.

\section{Acknowledgements}

We thank L-B Zhang, Q Zhu, Y-H Yang, H-Z Sun, H-Z Kong and other members of the Ge laboratory (Institute of Botany, CAS) and J Zhang (Beijing Genomics Institute) for technical assistance. We also thank $Z$ Yang (University College London, UK), BS Gaut (University of California at Irvine, USA), and L-B Zhang (Missouri Botanical Garden, USA) for discussions and suggestions. We are grateful to BR Holland (Massey University, New Zealand) for providing Python scripts for creating consensus networks and J Savard (University of Koln, Germany) for providing Perl scripts for gene bootstrapping. We acknowledge the International Rice Research Institute (Los Banos, Philippines) for providing seed samples. This work was supported by the National Basic Research Program of China (2007CB8I5704), the National Natural Science Foundation of China (30121003, 30430030, 30025005), and grants from the Chinese Academy of Sciences to GS, and the National Science Foundation of USA to TS.

\section{References}

I. Ge S, Sang T, Lu BR, Hong DY: Phylogeny of the genus Oryza as revealed by molecular approaches. In Rice Genetics IV Proceedings of the Fourth International Rice Genetics Symposium: 25 October, 2000; Los Baños, Laguna, Philippines Edited by: Khush GS, Brar DS, Hardy B. Los Banos, Philippines: International Rice Research Institute; 200I:89-105.

2. International Rice Genome Sequencing Project: The map-based sequence of the rice genome. Nature 2005, 436:793-800.

3. Kellogg EA: The grasses: a case study in macroevolution. Annu Rev Ecol Syst 2000, 3 I:2 17-238.

4. Shimamoto K, Kyozuka J: Rice as a model for comparative genomics of plants. Annu Rev Plant Biol 2002, 53:399-4I9.

5. Wing RA, Ammiraju JS, Luo M, Kim H, Yu Y, Kudrna D, Goicoechea JL, Wang W, Nelson W, Rao K, Brar D, Mackill DJ, Han B, Soderlund C, Stein L, SanMiguel P, Jackson S: The Oryza Map Alignment Project: the golden path to unlocking the genetic potential of wild rice species. Plant Mol Biol 2005, 59:53-62.

6. Bennetzen JL: Patterns in grass genome evolution. Curr Opin Plant Biol 2007, 10:176-181.

7. Ammiraju JS, Luo M, Goicoechea JL, Wang W, Kudrna D, Mueller C, Talag J, Kim H, Sisneros NB, Blackmon B, Fang E, Tomkins JB, Brar D, MacKill D, McCouch S, Kurata N, Lambert G, Galbraith DW, Arumuganathan K, Rao K, Walling JG, Gill N, Yu Y, SanMiguel P, Soderlund C, Jackson S, Wing RA: The Oryza bacterial artificial chromosome library resource: construction and analysis of 12 deepcoverage large-insert BAC libraries that represent the 10 genome types of the genus Oryza. Genome Res 2006, I 6: | $40-147$

8. Piegu B, Guyot R, Picault N, Roulin A, Saniyal A, Kim H, Collura K, Brar DS, Jackson S, Wing RA, Panaud O: Doubling genome size without polyploidization: dynamics of retrotranspositiondriven genomic expansions in Oryza australiensis, a wild relative of rice. Genome Res 2006, I6:1262-1269.

9. Vaughan DA: The genus Oryza L.: current status of taxonomy. IRRI Res Pap Ser 1989, I 38: I-2I.

10. Nayar NM: Origin and cytogenetics of rice. Adv Genet 1973, I 7:153-292.

II. Aggarwal RK, Brar DS, Khush GS: Two new genomes in the Oryza complex identified on the basis of molecular divergence analysis using total genomic DNA hybridization. Mol Gen Genet 1997, 254: I-12.

12. Ge S, Sang T, Lu BR, Hong DY: Phylogeny of rice genomes with emphasis on origins of allotetraploid species. Proc Natl Acad Sci USA 1999, 96: |4400-|4405.

13. Gong Y, Borromeo T, Lu BR: A biosystematic study of the Oryza meyeriana complex (Poaceae). Plant Syst Evol 2000, 224: I39-I 5 I.

14. Second G: A new insight into the genome differentiation in Oryza L. through isozymic studies. In Advances in Chromosome and Cell Genetics Edited by: Sharma AK, Sharma A. New Delhi: Oxford and IBH; 1985:45-78.

15. Wang ZY, Second G, Tanksley SD: Polymorphism and phylogenetic relationships among species in the genus Oryza as determined by analysis of nuclear RFLPs. Theor Appl Genet 1992, 83:565-58|.

16. Mullins $M$, Hilu W: Sequence variation in the gene encoding the I0-kDa prolamin in Oryza (Poaceae). I. Phylogenetic implications. Theor Appl Genet 2002, 105:84I-846.

17. Sharma SD: Evolutionary trends in genus Oryza. In Rice Genetics 
Los Banos, Philippines: International Rice Research Institute; 1986:59-67.

18. Joshi SP, Gupta VS, Aggarwal RK, Ranjekar PK, Brar DS: Genetic diversity and phylogenetic relationship as revealed by inter simple sequence repeat (ISSR) polymorphism in the genus Oryza. Theor Appl Genet 2000, I00:13 I I-1320.

19. Zhang SH, Second G: Phylogenetic analysis of the tribe Oryzeae: total chloroplast DNA restriction fragment analysis (a preliminary report). Rice Genet Newsl 1989, 6:76-80.

20. Zhang Q, Kochert G: Independent amplification of two classes of Tourists in some Oryza species. Genetica 1997, I0I:I45-152.

2I. Bapteste E, Brinkmann H, Lee JA, Moore DV, Sensen CW, Gordon P, Durufle L, Gaasterland T, Lopez P, Muller M, Philippe H: The analysis of 100 genes supports the grouping of three highly divergent amoebae: Dictyostelium, Entamoeba, and Mastigamoeba. Proc Natl Acad Sci USA 2002, 99: 1414-1419.

22. Rokas A, Williams BL, King N, Carroll SB: Genome-scale approaches to resolving incongruence in molecular phylogenies. Nature 2003, 425:798-804.

23. Dopazo H, Dopazo J: Genome-scale evidence of the nematodearthropod clade. Genome Biol 2005, 6:R4I

24. Pfeil BE, Schlueter JA, Shoemaker RC, Doyle JJ: Placing paleopolyploidy in relation to taxon divergence: a phylogenetic analysis in legumes using 39 gene families. Syst Biol 2005, 54:44 I-454.

25. Rokas A, Kruger D, Carroll SB: Animal evolution and the molecular signature of radiations compressed in time. Science 2005, 310:1933-1938.

26. Pollard DA, lyer VN, Moses AM, Eisen MB: Widespread discordance of gene trees with species tree in Drosophila: evidence for incomplete lineage sorting. PLoS Genet 2006, 2:e 173.

27. Savard J, Tautz D, Richards S, Weinstock GM, Gibbs RA, Werren JH, Tettelin H, Lercher MJ: Phylogenomic analysis reveals bees and wasps (Hymenoptera) at the base of the radiation of Holometabolous insects. Genome Res 2006, 16:1334-1338.

28. Rodriguez-Ezpeleta N, Brinkmann H, Roure B, Lartillot N, Lang BF, Philippe $\mathrm{H}$ : Detecting and overcoming systematic errors in genome-scale phylogenies. Syst Biol 2007, 56:389-399.

29. Rasmussen MD, Kellis M: Accurate gene-tree reconstruction by learning gene- and species-specific substitution rates across multiple complete genomes. Genome Res 2007, 17:1932-1942.

30. Nishihara H, Okada N, Hasegawa M: Rooting the eutherian tree: the power and pitfalls of phylogenomics. Genome Biol 2007, 8:RI99.

31. Delsuc F, Brinkmann H, Philippe H: Phylogenomics and the reconstruction of the tree of life. Nat Rev Genet 2005, 6:36I-375.

32. Philippe H, Delsuc F, Brinkmann H, Lartillot N: Phylogenomics. Annu Rev Ecol Evol Syst 2005, 36:54I-562.

33. Rokas A, Carroll SB: Bushes in the tree of life. PLoS Biol 2006, 4:e352.

34. Phillips MJ, Delsuc F, Penny D: Genome-scale phylogeny and the detection of systematic biases. Mol Biol Evol 2004, 2 I : | 455- I 458.

35. Jeffroy O, Brinkmann H, Delsuc F, Philippe H: Phylogenomics: the beginning of incongruence? Trends Genet 2006, 22:225-23I.

36. Lockhart PJ, Steel MA, Hendy MD, Penny D: Recovering evolutionary trees under a more realistic model of sequence evolution. Mol Biol Evol 1994, I I:605-6 I2.

37. Tajima F: Simple methods for testing the molecular evolutionary clock hypothesis. Genetics 1993, 135:599-607.

38. Woese CR, Achenbach L, Rouviere P, Mandelco L: Archaeal phylogeny: reexamination of the phylogenetic position of Archaeoglobus fulgidus in light of certain composition-induced artifacts. Syst Appl Microbiol I99I, 14:364-37I.

39. Zhao W, Wang J, He X, Huang X, Jiao Y, Dai M, Wei S, Fu J, Chen $Y$, Ren X, Zhang Y, Ni P, Zhang J, Li S, Wang J, Wong GK, Zhao H, Yu J, Yang $\mathrm{H}$, Wang J: BGI-RIS: an integrated information resource and comparative analysis workbench for rice genomics. Nucleic Acids Res 2004, 32:D377-382.

40. de Queiroz A, Donoghue MJ, Kim J: Separate versus combined analysis of phylogenetic evidence. Annu Rev Ecol Syst 1995, 26:657-68I.

41. Doyle J]: Trees within trees: genes and species, molecules and morphology. Syst Biol 1997, 46:537-553.

42. Linder CR, Rieseberg LH: Reconstructing patterns of reticulate evolution in plants. Am J Bot 2004, 91 : 1700-1708.

43. Pamilo $P$, Nei M: Relationships between gene trees and species trees. Mol Biol Evol 1988, 5:568-583.

44. Maddison WP: Gene trees in species trees. Syst Biol 1997, 46:523-536.
45. Zhang LB, Ge S: Multilocus analysis of nucleotide variation and speciation in Oryza officinalis and its close relatives. Mol Biol Evol 2007, 24:769-783.

46. Zhu Q, Zheng X, Luo J, Gaut BS, Ge S: Multilocus analysis of nucleotide variation of Oryza sativa and its wild relatives: severe bottleneck during domestication of rice. Mol Biol Evol 2007, 24:875-888.

47. Gaut BS, Morton BR, McCaig BC, Clegg MT: Substitution rate comparisons between grasses and palms: synonymous rate differences at the nuclear gene $A d h$ parallel rate differences at the plastid gene rbcL. Proc Natl Acad Sci USA 1996, 93:10274-10279.

48. White SE, Doebley JF: The molecular evolution of terminal earl, a regulatory gene in the genus Zea. Genetics 1999, 153:1455-1462

49. Zhu Q, Ge S: Phylogenetic relationships among A-genome species of the genus Oryza revealed by intron sequences of four nuclear genes. New Phytol 2005, 167:249-265.

50. Vaughan DA: The Wild Relatives of Rice: a Genetic Resources Handbook Los Banos, Philippines: International Rice Research Institute; 1994.

5I. Whitfield JB, Lockhart PJ: Deciphering ancient rapid radiations. Trends Ecol Evol 2007, 22:258-265.

52. Enard W, Paabo S: Comparative primate genomics. Annu Rev Genom Hum Genet 2004, 5:35I-378.

53. Guo YL, Ge S: Molecular phylogeny of Oryzeae (Poaceae) based on DNA sequences from chloroplast, mitochondrial, and nuclear genomes. Am J Bot 2005, 92:1548-1558.

54. Baldwin BG, Sanderson MJ: Age and rate of diversification of the hawaiian silversword alliance (Compositae). Proc Natl Acad Sci USA 1998, 95:9402-9406.

55. Wendel JF, Doyle J]: Phylogenetic incongruence: window into genome history and molecular evolution. In Molecular Systematics of Plants II: DNA Sequencing Edited by: Soltis DE, Soltis PS, Doyle II. Boston: Kluwer; 1998:265-296.

56. Fishbein M, Hibsch-Jetter C, Soltis DE, Hufford L: Phylogeny of Saxifragales (angiosperms, eudicots): analysis of a rapid, ancient radiation. Syst Biol 200I, 50:8I7-847.

57. Chen FC, Li WH: Genomic divergences between humans and other hominoids and the effective population size of the common ancestor of humans and chimpanzees. Am J Hum Genet 200I, 68:444-456.

58. Patterson N, Richter DJ, Gnerre S, Lander ES, Reich D: Genetic evidence for complex speciation of humans and chimpanzees. Nature 2006, 44 I: I I03-I I08.

59. Takezaki N, Figueroa F, Zaleska-Rutczynska Z, Takahata N, Klein J: The phylogenetic relationship of tetrapod, coelacanth, and lungfish revealed by the sequences of forty-four nuclear genes. Mol Biol Evol 2004, 21: I5I2-I524.

60. Qiu YL, Li L, Wang B, Chen Z, Knoop V, Groth-Malonek M, Dombrovska O, Lee J, Kent L, Rest J, Estabrook GF, Hendry TA, Taylor DW, Testa CM, Ambros M, Crandall-Stotler B, Duff RJ, Stech M, Frey $W$, Quandt $D$, Davis CC: The deepest divergences in land plants inferred from phylogenomic evidence. Proc Natl Acad Sci USA 2006, 103:155II-15516.

61. Walsh HE, Kidd MG, Moum T, Friesen VL: Polytomies and the power of phylogenetic inference. Evolution 1999, 53:932-937.

62. Wortley AH, Rudall PJ, Harris DJ, Scotland RW: How much data are needed to resolve a difficult phylogeny? Case study in Lamiales. Syst Biol 2005, 54:697-709.

63. Cummings MP, Otto SP, Wakeley J: Sampling properties of DNA sequence data in phylogenetic analysis. Mol Biol Evol 1995, | 2:8|4-822

64. Yu J, Wang J, Lin W, Li S, Li H, Zhou J, Ni P, Dong W, Hu S, Zeng C, Zhang J, Zhang Y, Li R, Xu Z, Li S, Li X, Zheng H, Cong L, Lin L, Yin J, Geng J, Li G, Shi J, Liu J, Lv H, Li J, Wang J, Deng Y, Ran L, Shi X, et al.: The genomes of Oryza sativa: a history of duplications. PLOS Biol 2005, 3:e38.

65. Kikuchi S, Satoh K, Nagata T, Kawagashira N, Doi K, Kishimoto N, Yazaki J, Ishikawa M, Yamada $\mathrm{H}$, Ooka $\mathrm{H}$, Hotta I, Kojima K, Namiki T, Ohneda E, Yahagi W, Suzuki K, Li C], Ohtsuki K, Shishiki T, Otomo Y, Murakami K, lida Y, Sugano S, Fujimura T, Suzuki Y, Tsunoda Y, Kurosaki T, Kodama T, Masuda H, Kobayashi M, et al.: Collection, mapping, and annotation of over 28,000 cDNA clones from japonica rice. Science 2003, 30 I:376-379.

66. Philippe H, Snell EA, Bapteste E, Lopez P, Holland PW, Casane D: Phylogenomics of eukaryotes: impact of missing data on large alignments. Mol Biol Evol 2004, 2 I: 1740-1752.

67. Notredame C, Higgins DG, Heringa J: T-Coffee: a novel method 
for fast and accurate multiple sequence alignment. I Mol Biol 2000, 302:205-217.

68. Swofford DL: PAUP*: Phylogenetic Analysis Using Parsimony (*and Other Methods) Sunderland, MA: Sinauer; 2002.

69. Felsenstein J: Confidence limits on phylogenies: an approach using the bootstrap. Evolution 1985, 39:783-79|.

70. Ronquist F, Huelsenbeck JP: MrBayes 3: Bayesian phylogenetic inference under mixed models. Bioinformatics 2003, 19:1572-1574.

71. Posada D, Crandall KA: MODELTEST: testing the model of DNA substitution. Bioinformatics 1998, I4:817-818.

72. Kass RE, Raftery AE: Bayes factors. I Am Stat Assoc 1995, 90:773-795.

73. Newton MA, Raftery AE, Davison AC, Bacha M, Celeux G, Carlin BP, Clifford P, Lu C, Sherman M, Tanner MA, Gelfand AE, Mallick BK, Gelman A, Grieve AP, Kunsch HR, Leonard T, Hsu JSJ, Liu JS, Rubin DB, Lo AY, Louis TA, Neal RM, Owen AB, Tu DS, Gilks WR, Roberts G, Sweeting T, Bates D, Ritter G, Worton BJ, et al.: Approximate Bayesian inference with the weighted likelihood bootstrap. J Roy Stat Soc Ser B (Stat Method) 1994, 56:3-48.

74. Huson DH, Bryant D: Application of phylogenetic networks in evolutionary studies. Mol Biol Evol 2006, 23:254-267.

75. Holland BR, Huber KT, Moulton V, Lockhart PJ: Using consensus networks to visualize contradictory evidence for species phylogeny. Mol Biol Evol 2004, 2 I: |459- | 46 |.

76. Holland BR, Jermiin LS, Moulton V: Improved consensus network techniques for genome-scale phylogeny. Mol Biol Evol 2006, 23:848-855

77. Huber KT, Langton M, Penny D, Moulton V, Hendy M: Spectronet: a package for computing spectra and median networks. Appl Bioinformatics 2002, I: I59-161.

78. Engstrom TN, Shaffer HB, McCord WP: Multiple data sets, high homoplasy, and the phylogeny of softshell turtles (Testudines: Trionychidae). Syst Biol 2004, 53:693-7IO.

79. Farris JS, Kallersjo M, Kluge AG, Bult C: Testing significance of incongruence. Cladistics 1994, 10:315-319.

80. Bull J, Huelsenbeck JP, Cunningham CW, Swofford DL, Waddell PJ: Partitioning and combining data in phylogenetic analysis. Syst Biol 1993, 42:384-397. 